\title{
The Effect of Mobility Models and Traffic Patterns on the Performance of Routing Protocols in MANETs
}

\author{
Mohamed Wahed \\ Faculty of Computers \\ \& Information, \\ Suez Canal \\ University, \\ Suze Canal, Egypt
}

\author{
Hassan Al-Mahdi \\ Faculty of Arts \& \\ Sciences, \\ AlJouf University, \\ Al Quryyat, Saudi \\ Arabia
}

\author{
Tarek M \\ Mahmoud \\ Faculty of Computers \\ \& Information, \\ Minia University, \\ Minia, Egypt
}

\author{
Hassan Shaban \\ Faculty of Computers \\ \& Information, \\ Minia University, \\ Minia, Egypt
}

\begin{abstract}
Mobile ad-hoc networks (MANETs) are self-organizing networks which can form a communication network without any fixed infrastructure. Constant bit rate (CBR) traffic pattern is very well known traffic model for MANETs which generates data packets at a constant rate. Transmission Control Protocol (TCP) provides reliability to data transferring in all end-to-end data stream services on the MANETs. There are several TCP traffic patterns such as TCP Reno, TCP New Reno, TCP Vegas, and TCP Selective Acknowledgment (Sack). The traffic pattern plays an important role in so far as the performance of a routing protocol is concerned. In this paper, we study the effect of impact of mobility models and traffic patterns on the behavior of Reactive (AODV) and Proactive (DSDV, OLSR) routing protocols used in MANETs considering both CBR and TCP traffic patterns with different mobility models namely, Reference Point Group Mobility (RPGM) and Manhattan Grid (MG). The performance metrics used to evaluate the efficiency of the considered protocols are packet delivery ratio, average throughput and End-to-End Delay. The experimental results conducted using NS2 simulator show that the relative ranking of routing protocols may vary depending on both mobility models and traffic patterns.
\end{abstract}

\section{Keywords}

MANETs; TCP traffic pattern; CBR traffic; Routing protocols; Manhattan Grid; Reference Point Group Mobility Model.

\section{INTRODUCTION}

Mobile ad hoc network (MANET) is a wireless network without infrastructure. Two or more mobile nodes can communicates each other directly if they are present within their transmission range. Otherwise, source node uses several number of intermediate nodes between the source and destination to relay the transmission. There is no centralized control among the mobile nodes. Hence, the routing and the resource management are maintained through diff erent nodes in distributed manner [1]. Routing protocols are categorized as reactive (AODV, DSR, AOMDV) and proactive protocols (DSDV, OLSR). The movement of mobile nodes may lead to breakup of communication links between source and destination [2]. The statistical behavior of physical movement of mobile nodes are described by diff erent mobility models like Random Way Point (RWP) [3, 4], Reference Point Group Mobility (RPGM) [6], Manhattan Grid (MG) [5] and GaussMarkov (GM) [7]. A survey of diverse array of mobility models is introduced in [8]. On the other hand, the traffic pattern affect the performance of the routing protocols. There exist two patterns of traffic, namely Constant Bit Rate (CBR) and Transmission Control Protocol (TCP). The CBR traffic makes no attempt to detect if the destination receives the transmitted data. It implies that data are sent at a fixed bit rate. TCP is connection-oriented and reliable delivery protocol, described in RFC 793 [9]. It is observed that most of the research investigations are based on CBR traffic pattern whereas most of the traffic on the Internet carries TCP. Thus, the study of TCP performance in MANET has become an important area of contemporary research [10]. There are several TCP patterns such as TCP Reno, TCP New Reno, TCP Vegas, and TCP Selective Acknowledgment (Sack). One of the purposes of this paper is studying and investigating the performance analysis of both reactive protocol (AODV) and proactive protocols (DSDV, OLSR) using both CBR and TCP traffic patterns under different mobility models. The remainder of this paper is organized as follows. Section 2 introduces a brief overview of related works. Section 3 contains the simulation settings and results. Finally, we summarize and conclude our paper in section 4 .

\section{RELATED WORKS}

Vikas s. and Parveen K. [10] presented a study conducted to evaluate the performance of Reactive (AODV, Temporally ordered routing algorithm (TORA)) and Proactive protocols (DSDV) of MANET based on both CBR and TCP traffic. These routing protocols were compared in terms of Packet delivery ratio, average end-to end delay and throughput when subjected to change in number of nodes. Simulation results show that Reactive protocols better in terms of packet delivery ratio and average end-to-end delay. In case of CBR traffic, throughput remains almost constant for all three protocols irrespective of number of nodes. In case of TCP traffic, throughput changes rapidly with respect to change in the number of nodes. Reactive protocols perform better than the proactive protocols in case of CBR traffic pattern. In the case of TCP traffic pattern, Packet delivery ratio of AODV protocols remains almost constant whereas it changes rapidly for TORA and DSDV protocols irrespective of the network load.

Muhammad I. and Nasir N. [11] analyzed the characteristics of Dynamic MANET on demand routing (DYMO) protocol by varying traffic patterns (CBR and TCP), number of nodes and topological areas. DYMO showed ups and downs in Packet Delivery Ratio using either traffic patterns, especially using CBR traffic. In case of packet delivery ratio, DYMO is better suited to TCP traffic than CBR. However, DYMO 
performs poorly in terms of Normalized Routing Load and Routing Overhead using either traffic patterns. The change of speed has more impact on DYMO, due to the fact that rapid change in topology causes change in node links and routing tables.

Patil V.P. [12] studied the performance of two MANET protocols AODV and DSDV based on TCP and CBR traffic. These routing protocols were compared in terms of packet delivery ratio, average end-to-end delay, and throughput when subjected to change in number of nodes and traffic pattern. Simulation results showed that reactive protocols were better in terms of packet delivery ratio and average end-to-end delay.

Arindrajit P. et al. [13] tried to analyze the behavior of the mobile nodes for different speed for three different traffic patterns namely CBR, Exponential and Pareto. They found that AODV routing performs much better than DSR in Exponential and Pareto traffic. The Packet Delivery Ratio (PDR) for AODV routing remains same across all traffic patterns. The Normalized Routing Load (NRL) increases in DSR. The throughput decreases for increasing the node speed of all the traffic models in AODV and DSR routing.

Youssef S. et al. [14] found that the performance parameters of the routing protocols may vary depending on network load, mobility and network size. Under Manhattan Grid mobility Model, AODV and DSR experience the highest Packet Delivery Fraction and Throughput with the increase of nodes pause time, CBR traffic sources and mobile nodes number. However, DSDV experiences the lowest average end-to-end delay. AODV has a slightly higher Average end-to-end Delay than DSR.

Deepti V. and Deepika C. [15] evaluated the performance of the AODV routing protocol. They considered packet delivery fraction and average end-to-end delay as criteria for evaluating the performance of this protocol in the case CBR and TCP traffic patterns with connections for different pause time. The results of this paper showed that using CBR traffic is not a good indicator for the AODV protocol performance when subject to TCP traffic. Finally the TCP is better than CBR traffic.

\section{SIMULATION RESULTS AND ANALYSIS}

As we mentioned above, the goal of this paper is to study the effect of traffic patterns and mobility models on the performance of the considered protocols. Throughout the conducted experiments we measure the performance metrics by varying the node movement speed. The Bonn-Motion tool [14] is used to generate the considered mobility models with different node speeds $(10,20,30,40$ and $50 \mathrm{~m} / \mathrm{s})$. The movement area is assumed to be 1200 meter by 1200 meter square region. The number of mobile nodes that move in the movement area is set to 50 mobile nodes. NS-2 simulator version 2.35 [15] is used in the simulation process. We assume that the movement period of the mobile nodes within the movement area is set to $600 \mathrm{sec}$. To generate TCP-Reno, TCP-Newreno, TCP-Vegas, and TCP-Sack traffic we modify cbrgen tool, which is a part of NS-2 simulator.

\subsection{Performance Metrics}

The following performance metrics are considered to analyze the performance of routing protocols under CBR and various TCP traffic types and variant mobility models.
Packet delivery ratio $D \%$ can be defined as the ratio of number data packets $P_{S}$ send to the destination to the number of data packets $P_{r}$ received by the destination. Hence the value of $D$ can be written as follows.

$$
D \%=\frac{P_{s}}{P_{r}} x 100 .
$$

The Average Throughput $T$ can be defined as the ratio of the total number of packets flow $P_{f}$ in the network to the simulation time $\mathrm{S}$. That is,

$$
T=\frac{P_{f}}{S}
$$

The Average End-to-End Delay is defined as the average time taken for an entire message to completely arrive at the destination from the source. Evaluation of end-to-end delay mostly depends on the following components: propagation time (PT), transmission time (TT), queuing time (QT) and processing delay (PD). It is evaluated as:

$$
\text { End }- \text { to }- \text { End Delay }=P T+T T+Q T+P D
$$

In the simulation process, 75 random scenarios are generated by 75 simulation run for each considered protocol. Table 1 shows the main characteristics used for scenarios.

Table 1. The main simulation parameters used for scenarios

\begin{tabular}{|c|c|}
\hline Simulation parameters & Value \\
\hline Simulator & NS2 Version 2.35 \\
\hline MAC Type & W02.11 \\
\hline Channel type & TwoRayGround \\
\hline $\begin{array}{c}\text { Radio Propagation } \\
\text { Model }\end{array}$ & 50 \\
\hline Interface Queue Length & DropTail/PriQueue \\
\hline Interface Queue Type & AODV, DSDV, OLSR \\
\hline Protocol studied & $1200 \mathrm{~m}$ x $1200 \mathrm{~m}$ \\
\hline Simulation area & 50 \\
\hline Number of Nodes & $10,20,30,40,50 \mathrm{~m} / \mathrm{s}$ \\
\hline Node speed & CBR/UDP, ftp/TCP (TCP-Reno, \\
\hline Traffic pattern & TCP-Newreno, TCP-Vegas, TCP- \\
\hline Mobility model & RPGM, MG \\
\hline Pause time & 0s (random) \\
\hline Simulation time & 600 c \\
\hline \multicolumn{2}{|c}{}
\end{tabular}

\subsection{Performance Analysis in RPGM Mobility Model}

The following subsections illustrate the effect of RPGM mobility model and the used traffic patterns on the performance of the considered protocols. We realize simulation for 50 nodes. The nodes are divided into 5 groups of size 10 nodes for each.

\subsubsection{Packet Delivery Ratio}

Figures 1a-1c illustrate packet delivery ratio D\% versus nodes movement speed for the considered protocols with CBR and TCP traffic patterns (TCP-Reno, TCP-Newreno, TCP-Vegas and TCP-Sack). The Figures show that in the case of CBR traffic, the packet delivery ratio $\mathrm{D} \%$ of $\mathrm{AODV}$ is increasing as the node movement speed is increasing (over $30 \mathrm{~m} / \mathrm{s}$ ). Also, the D\% of AODV is better than both DSDV and OLSR protocols. The $\mathrm{D} \%$ of the considered protocols in the case of TCP traffic is better compared to the $\mathrm{D} \%$ of the considered 
protocols in the case of CBR traffic pattern. The D\% in the case of TCP-Vegas traffic pattern is the best for all considered routing protocols and all considered traffic patterns.

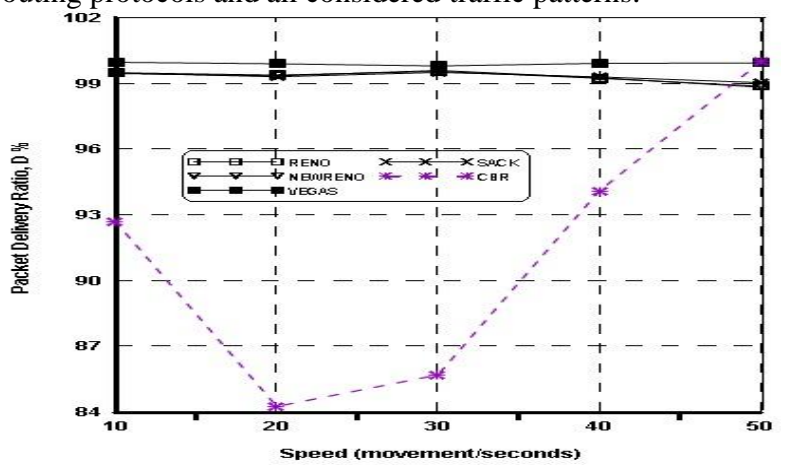

Fig 1a: Packet Delivery Ratio D \% in AODV routing protocol with RPGM mobility for different node movement speed using CBR and different TCP traffic patterns

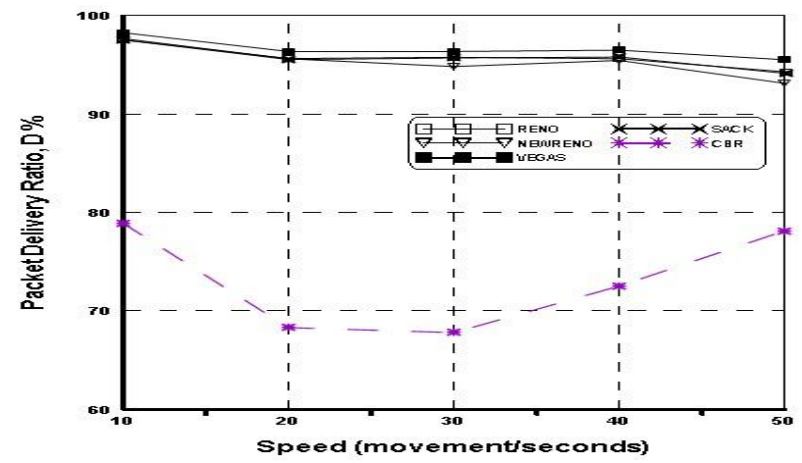

Fig 1b: Packet Delivery Ratio D \% in DSDV routing protocol and RPGM mobility versus node movement speed with CBR and different TCP traffic patterns

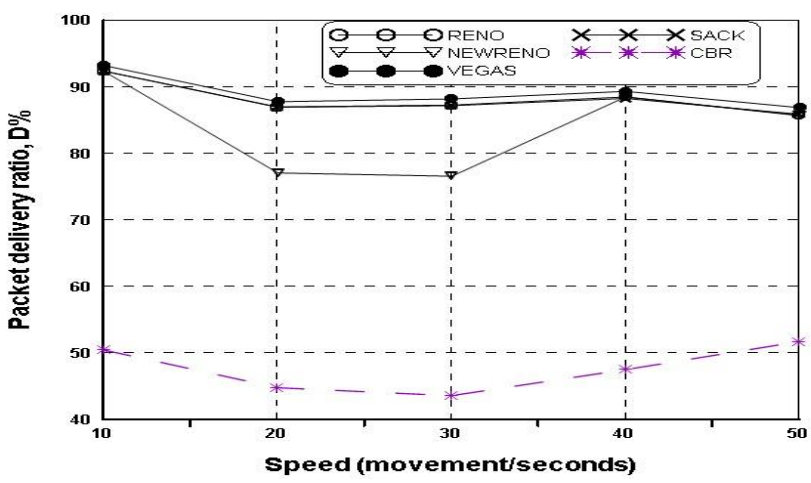

Fig 1c: Packet Delivery Ratio D \% in OLSR routing protocol with RPGM mobility for different node movement speed using CBR and different TCP traffic patterns.

\subsubsection{Average Throughput}

Figures $2 \mathrm{a}-2 \mathrm{c}$ indicate the average throughput $\mathrm{T}$ for the considered protocols with $\mathrm{CBR}$ and different TCP traffic patterns versus the nodes movement speed. The Figures show that, the average throughput $\mathrm{T}$ has lowest values and almost stable for all considered protocols with CBR traffic pattern irrespective of the mobile node speed. In the case of TCP traffic, the $\mathrm{T}$ values are changing up and down as the node movement speed is increasing for the considered routing protocols. The average throughput of DSDV and OLSR protocols is better than AODV protocol.

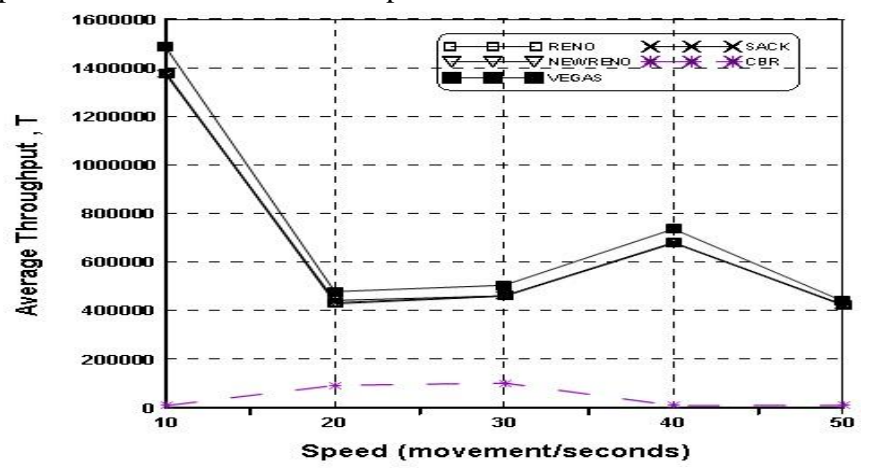

Fig 2a: Average Throughput $\mathrm{T}$ of AODV routing protocol with RPGM mobility for different node movement speed using CBR and different TCP traffic patterns.

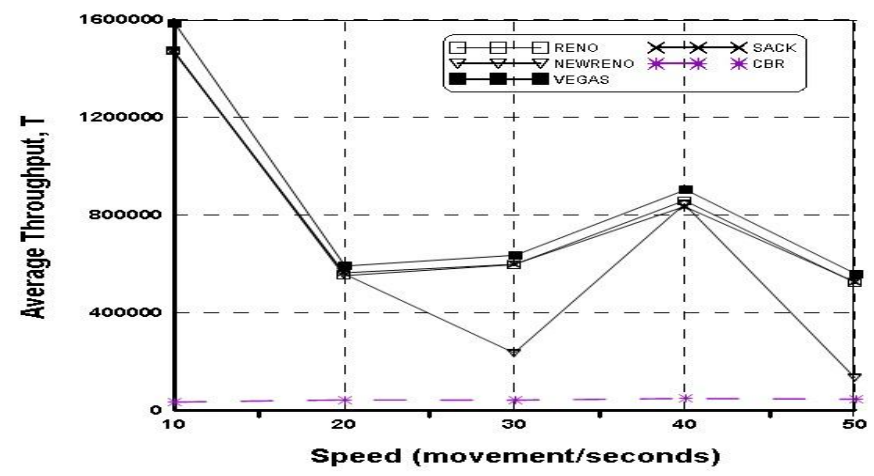

Fig 2b: Average Throughput $\mathrm{T}$ in DSDV routing protocol with RPGM mobility for different node movement speed using CBR and different TCP traffic patterns.

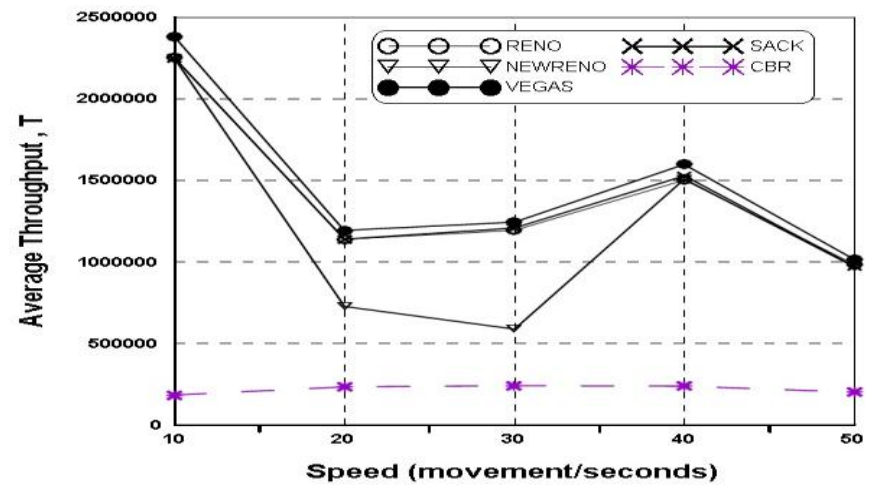

Fig 2c: Average Throughput $\mathrm{T}$ in OLSR routing protocol with RPGM mobility for different node movement speed using CBR and different TCP traffic patterns.

\subsubsection{Average End-to-End Delay}

Figures 3a-3c demonstrate the dependence of average end-toend delay on the node movement speed for different routing protocols and with both CBR and different TCP types. As graphs show, in case of CBR traffic, the average end to end delay values is low for all considered routing protocols as compared to TCP traffic pattern. All considered routing protocols produce lowest average End-to-End delay with TCPVegas compared to other considered TCP traffic patterns. 


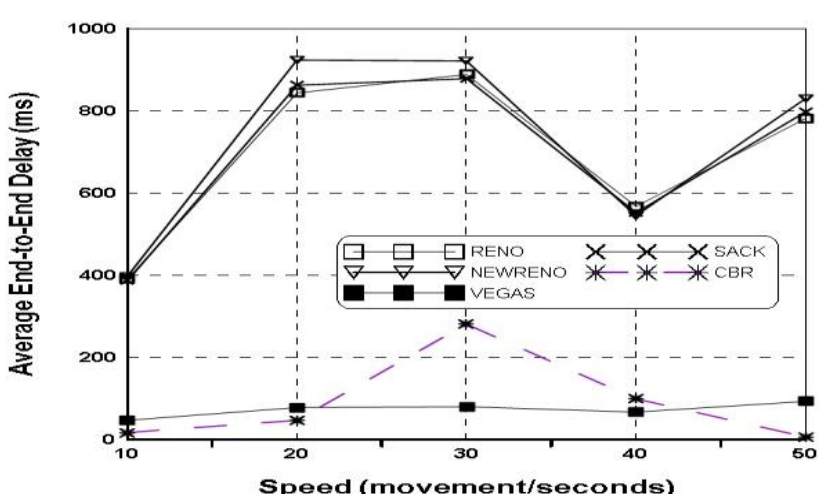

Fig 3a: Average End-to-End Delay of AODV routing protocol with RPGM mobility for different node movement speed using CBR and different TCP traffic patterns.

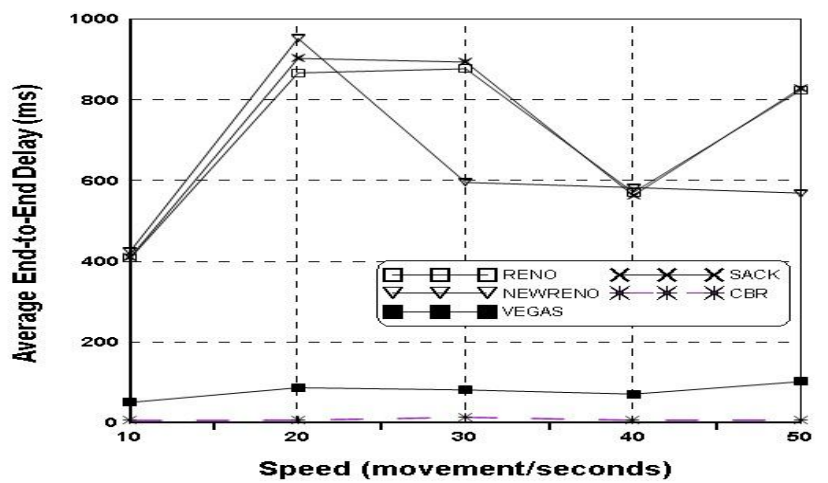

Fig 3b: Average End-to-End Delay of DSDV routing protocol with RPGM mobility for different node movement speed using CBR and different TCP traffic patterns.

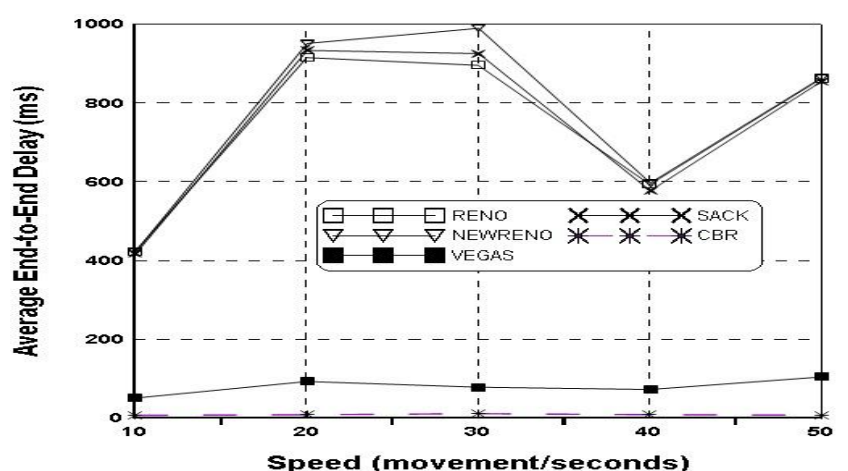

Fig 3c: Average End-to-End Delay of OLSR routing protocol with RPGM mobility for different node movement speed using CBR and different TCP traffic patterns

The average simulation results of the considered performance metrics for AODV, DSDV and OLSR indicate that, the D\% and average throughput $\mathrm{T}$ are better in the case of TCP-Vegas traffic pattern compared with $\mathrm{CBR}$ and other TCP traffic patterns. Considering the end-to-end delay metric, the performance of the DSDV and OLSR protocols are better in the case of CBR traffic pattern compared with the considered TCP traffic patterns. The end-to-end delay of AODV protocol is better in the case of TCP-Vegas compared with CBR and other TCP traffic patterns.

\subsection{Performance Analysis in MG Mobility Model}

In this section the effect of MG mobility model on the considered routing protocols with different traffic patterns is evaluated.

\subsubsection{Packet Delivery Ratio}

Figures $6 a-6 \mathrm{c}$ show the relation between the node movement speed and packet delivery ratio $\mathrm{d} \%$. In the case of CBR traffic, the packet delivery ratio $\mathrm{D} \%$ values are low for all considered routing protocols compared to TCP traffic patterns. It can been seen also that, the $\mathrm{D} \%$ of AODV routing protocol is higher than both DSDV and OLSR protocols. In the case of TCP-Vegas traffic pattern, The $\mathrm{D} \%$ of AODV routing protocol has higher values compared with TCP-Reno, TCP Newreno, and TCP-Sack traffic patterns. Considering DSDV and OLSR routing protocols, the $\mathrm{D} \%$ values of DSDV are higher than OLSR for all TCP traffic patterns.

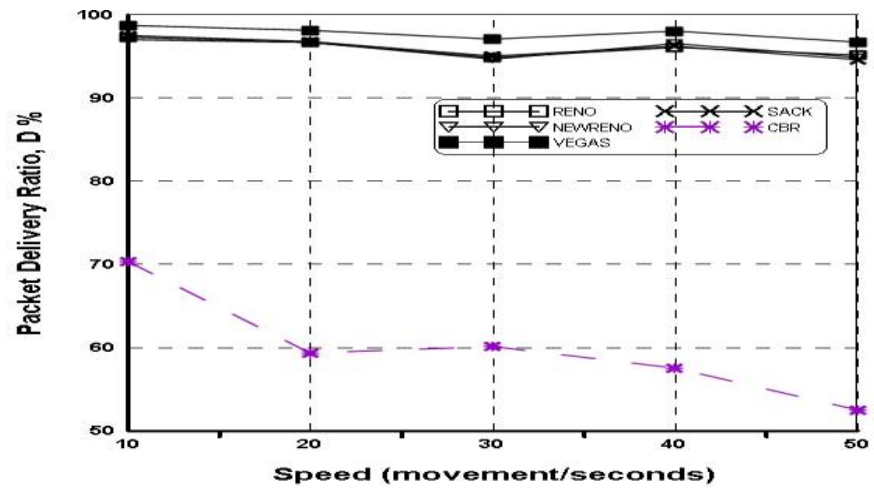

Fig 6a: Packet Delivery Ratio D\% of AODV routing protocol with MG mobility for different node movement speed using CBR and different TCP traffic patterns.

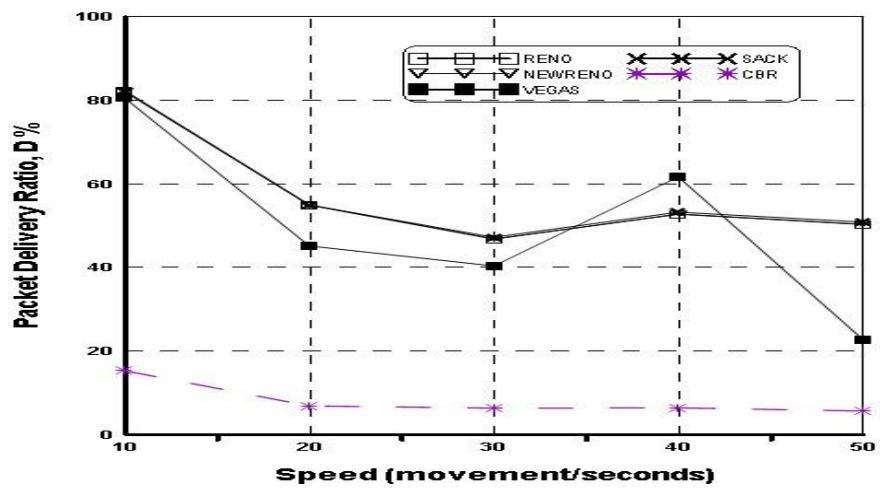

Fig 6b: Packet Delivery Ratio D\% of DSDV routing protocol with MG mobility for different node movement speed using CBR and different TCP traffic patterns. 


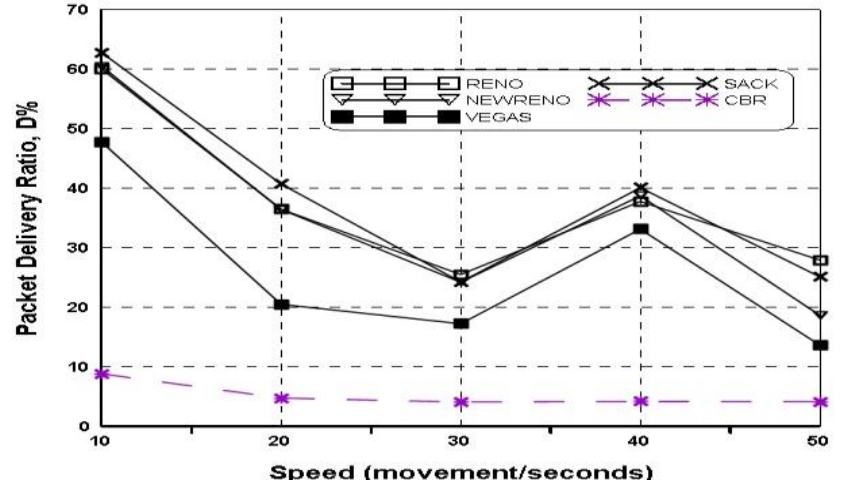

Fig 6c: Packet Delivery Ratio D\% of OLSR routing protocol with MG mobility for different node movement speed using CBR and different TCP traffic patterns.

\subsubsection{Average Throughput}

Figures $7 \mathrm{a}-7 \mathrm{c}$ represent the relation between the average throughput of the considered routing protocols with both CBR and TCP traffic patterns versus the nodes movement speed. As can been seen in these Figures, the average throughput $\mathrm{T}$ has lowest values for AODV protocol with CBR traffic pattern. Considering TCP traffic patterns the T values are changed rapidly for the considered routing protocols irrespective of the mobile nodes speed. The average throughput of OLSR protocol has higher values in the case of TCP-Sack compared with other TCP traffic patterns.

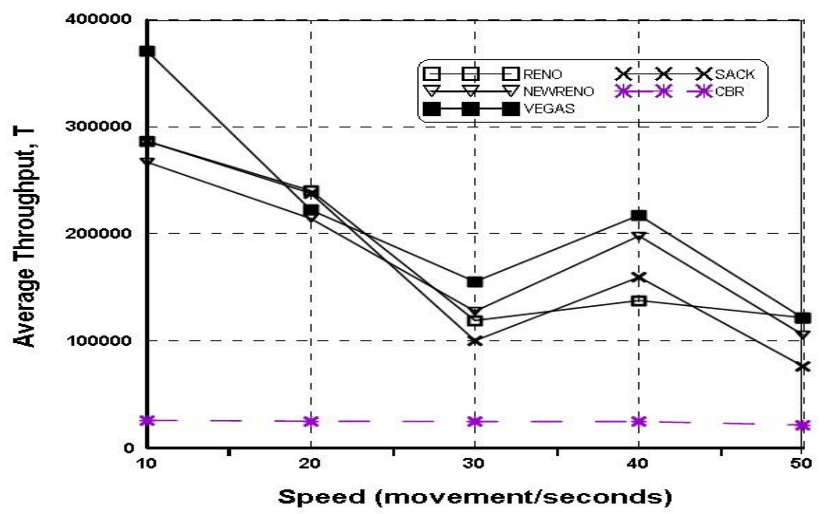

Fig 7a: Average Throughput T of AODV routing protocol with MG mobility for different node movement speed using CBR and different TCP traffic patterns.

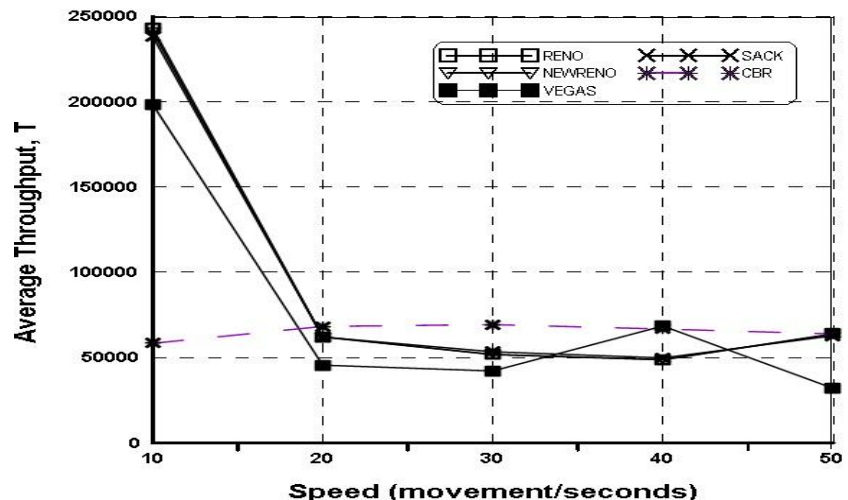

Fig 7b: Average Throughput $\mathrm{T}$ of DSDV routing protocol with MG mobility for different node movement speed using CBR and different TCP traffic patterns.

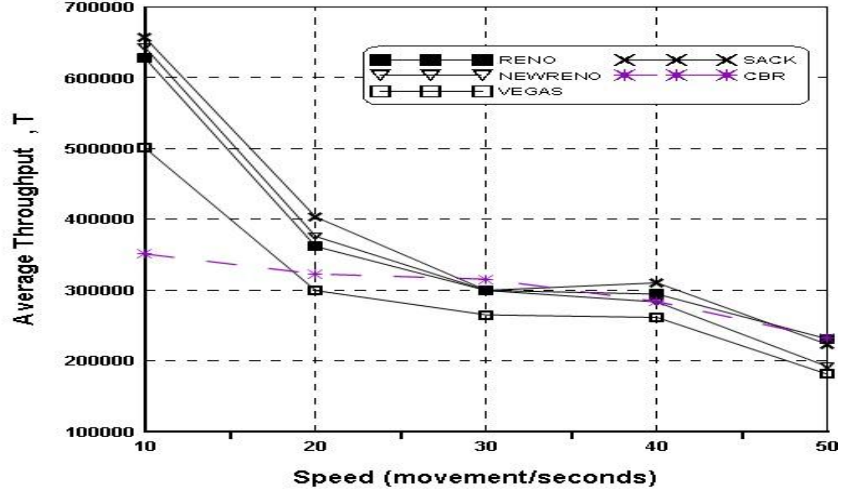

Fig 7c: Average Throughput T of OLSR routing protocol with MG mobility for different node movement speed using CBR and different TCP traffic patterns.

\subsubsection{Avaerage End-to-End Delay}

Figures 8a-8c show the performance metric Average End-toEnd Delay of the considered protocols with respect to the node movement speed in the case of CBR and TCP traffic patterns. As can been seen in these Figures the end-to-end delay of the considered routing protocols is high in the case of CBR traffic pattern compared with all TCP traffic patterns. Considering TCP traffic pattern, the end-to-end delay has lowest values in the case of TCP-Vegas traffic pattern for all the considered protocols.

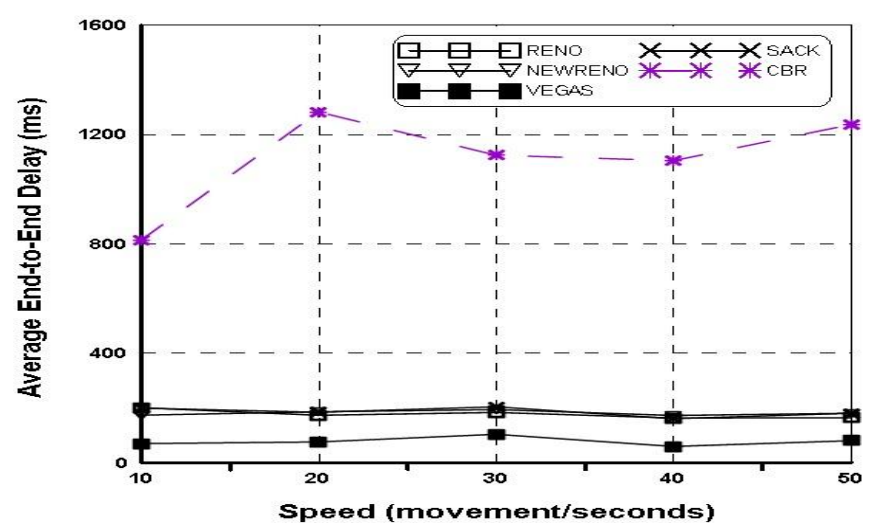

Fig 8a: Average End-to-End Delay of AODV routing protocol with MG mobility for different node movement speed using CBR and different TCP traffic patterns.

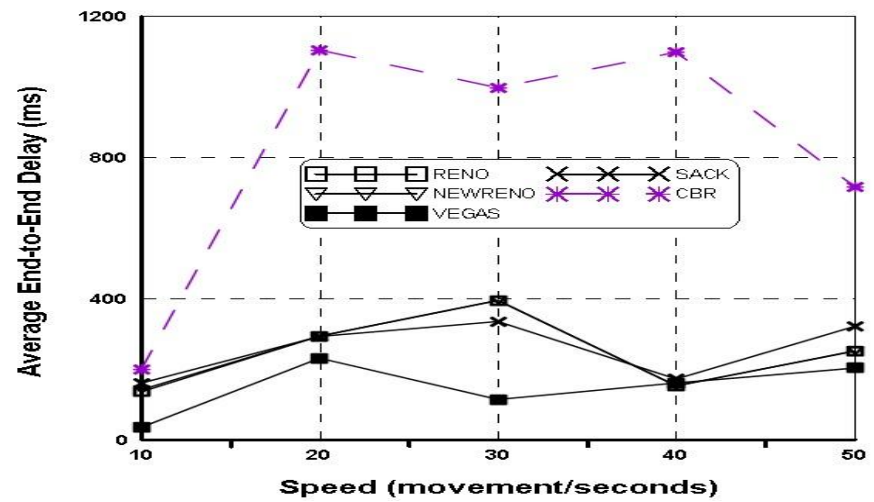

Fig 8b: Average End-to-End Delay of DSDV routing protocol with MG mobility for different node movement speed using CBR and different TCP traffic patterns. 


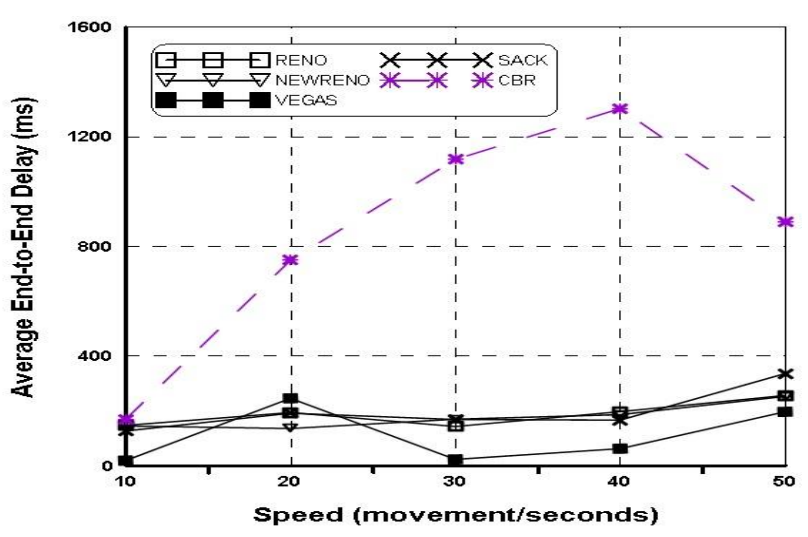

Fig 8c: Average End-to-End Delay of OLSR routing protocol with MG mobility for different node movement speed using CBR and different TCP traffic patterns.

The average simulation results of the considered performance metrics for AODV, DSDV and OLSR protocols in the case of MG mobility model indicate that, in the case of CBR traffic pattern, the $\mathrm{D} \%$ and average throughput $\mathrm{T}$ has the lowest values for the considered protocols compared with TCP traffic patterns. On the contrary the average End-To-End Delay has highest values. Considering TCP-Vegas traffic pattern the obtained results show that, D\% and average throughput of AODV protocol has higher values compared with other TCP traffic patterns. In case of TCP-Sack traffic pattern the $\mathrm{D} \%$ of both DSDV and OLSR protocols are the best. The average throughput T of DSDV in the case of TCP-Reno is the best, while the average throughput of OLSR is the best in the case of TCP-Sack traffic pattern. The end-to-end delay for the considered protocols is the best in case of TCP-Vegas traffic pattern compared to other TCP traffic patterns.

Table 2. Concludes all the obtained simulation results of the considered protocols for all mobility models. The $\mathrm{W}$ and $\mathrm{G}$ columns denote to the weak and the good performance metrics.

Table 2. The weak $\&$ good performance of $d \%$, average throughput T and end-to-end delay of AODV, DSDV and OLSR routing protocols versus the node movement speed in case of PRGM and MG mobility models

\begin{tabular}{|c|c|c|c|c|c|}
\hline \multirow{2}{*}{ Metrics } & \multirow{2}{*}{$\begin{array}{l}\text { Proto } \\
\text { cols }\end{array}$} & \multicolumn{2}{|c|}{ RPGM } & \multicolumn{2}{|c|}{ MG } \\
\hline & & $W$ & $G$ & $W$ & $G$ \\
\hline \multirow{3}{*}{ D\% } & $A O D V$ & CBR & Vegas & CBR & Vegas \\
\hline & $D S D V$ & CBR & Vegas & CBR & Sack \\
\hline & OLSR & CBR & Vegas & CBR & Sack \\
\hline \multirow{3}{*}{$\begin{array}{c}\text { Average } \\
\text { Throughput }\end{array}$} & $A O D V$ & CBR & Vegas & CBR & Vegas \\
\hline & $D S D V$ & CBR & Vegas & CBR & Sack \\
\hline & OLSR & CBR & Vegas & CBR & Sack \\
\hline \multirow{3}{*}{$\begin{array}{c}\text { Average } \\
\text { End-to-End } \\
\text { Delay }\end{array}$} & $A O D V$ & Vegas & Newreno & Vegas & CBR \\
\hline & $D S D V$ & CBR & Sack & Vegas & CBR \\
\hline & OLSR & CBR & Newreno & Vegas & CBR \\
\hline
\end{tabular}

\section{CONCLUSION}

In this paper, we have tried to analyze the behavior of proactive (AODV) and reactive (DSDV and OLSR) routing protocols with different node movement speed for both CBR and four different TCP traffic types namely, TCP-Reno, TCP-Newreno, TCP-Vegas and TCP-Sack with respect to RPGM and Manhattan Grid mobility models using NS-2 simulator. The performance metrics used in the evaluation process were packet delivery ratio, average throughput and average End-toEnd Delay. Simulation results have indicated that the relative ranking of routing protocols may vary depending on mobility model, node movement speed and the traffic patterns. The simulation results indicated that, AODV protocol performed significantly better than both DSDV and OLSR in the case of TCP-Vegas regardless of the movement speeds and mobility models. Generally, the performance metrics of the considered protocols were better in the case of TCP traffic patterns and all mobility models compared with CBR traffic pattern. Only, the end-to-end delay metric of DSDV and OLSR protocols was better in the case of CBR traffic pattern and RPGM mobility model. In Future work, we can extend this work to study the impact of group mobility models and node movement speed on multipath and multicast routing protocols used in MANETs.

\section{REFERENCES}

[1] S. Kumar S, T. Basavaraju and C. Puttamadappa, 2008 "Ad Hoc Mobile Wireless Networks: Principles, protocols and applications", Auerbach Publications.

[2] A. Pal, J. Singh, P. Dutta, P. Basu, D. Basu, 2011 "A study on the effect of traffic patterns on routing protocols in ad- hoc network following RPGM mobility model", in: International Conference on Signal Processing, Communication, Computing and Networking Technologies, ICSCCN 2011, pp. 233-237

[3] C. Bettstetter, 2003 "Topology properties of ad hoc networks with random waypoint mobility", ACM SIGMOBILE Mobile Computing and Communication Review 7 (3), pp. 50-52.

[4] J. P. Singh, P. Dutta, "Temporal modeling of node mobility in mobile ad hoc network", Journal of Computing and Information Technology18(1)(2010)1929.http://dx.doi.org/doi:10.2498/cit.1001412 doi:doi:10.2498/cit.1001412.

[5] S. Himabindu Pucha, Y. Hu,2007 "The performance impact of traffic patterns on routing protocols in mobile ad hoc networks", Computer Networks 51, pp. 35953616.

[6] J. P. Singh and P. Dutta, 2011"Temporal modeling of link characteristics in mobile ad-hoc network," journal of computing and information technology.

[7] M. Esquius, 2010 "Evaluation of MANET Routing Protocols in Realistic Environments".

[8] T. Camp, J. Boleng, V. Davies, A survey of mobility models for ad hoc network research, Wireless Communications and Mobile Computing (WCMC): Special issue on Mobile Ad Hoc Networking: Research, Trends and Applications 2 (5), pp. 483-502, 2002.

[9] Transmission Control Pprotcol Rfc793, https://www.ietf.org/rfc/rfc793.txt last visited Mar. 2014

[10] Vikas s., Parveen K., "Traffic Pattern based performance comparison of reactive and proactive protocols of mobile Ad-hoc Networks", International Journal of computer Application, Volume 5- No. 10, 2010.

[11] M. Inyat, and N. Nawaz, 2011 "Measuring the Effect of CBR and TCP Traffic Models over DYMO Routing Protocol", Global Journal of Computer Science and Technology Volume 11 Issue 14 Version 1.0 
[12] Patil V.P.,2012 "Effect of Traffic pattern on the Performance of Table Driven and On Demand Routing Protocols of MANET", International Journal Of Computational Engineering Research Vol. 2 Issue. 5, pp. $1311-1317$

[13] A. Pal, J. P. Singh, P. Duttac, 2012 "The Effect of speed variation on different Traffic Patterns in Mobile Ad Hoc Network" Elsiver, Procedia Technology 4, pp. 743 - 748
[14] Y. Saadi, S. El Kafhali, A. Haqiq., B. Nassereddine, 2012 "Simulation Analysis of Routing Protocols using Manhattan Grid Mobility Model in MANET", International Journal of Computer Applications Volume 45- No.23.

[15] D. Verma, D. Chandrawanshi,2011 "Comparative Performance Evaluation of AODV over CBR and TCP Traffic,” IJCST Vol. 2, Issue 2. 